OPEN ACCESS

Edited by:

Phuc Huu Phan,

Vietnam National Hospital of

Pediatrics, Vietnam

Reviewed by:

Ellen Stobberingh,

National Institute Public Health,

Netherlands

Gazi Arslan,

Dokuz Eylül University, Turkey

*Correspondence:

Suvash Chandra Ojha

suvash_ojha@swmu.edu.cn

Specialty section:

This article was submitted to

Pediatric Critical Care,

a section of the journal

Frontiers in Pediatrics

Received: 23 May 2021

Accepted: 12 July 2021

Published: 05 August 2021

Citation:

Chen K, Malik AA, Sheng Y-J, Ahmed S, Sun C, Deng C- $L$ and Ojha SC (2021) Clinical Utility of

Molecular Tests for Guiding Therapeutic Decisions in Bloodstream

Staphylococcal Infections: A Meta-Analysis.

Front. Pediatr. 9:713447.

doi: 10.3389/fped.2021.713447

\section{Clinical Utility of Molecular Tests for Guiding Therapeutic Decisions in Bloodstream Staphylococcal Infections: A Meta-Analysis}

\author{
Ke Chen 1,2, Aijaz Ahmad Malik ${ }^{3}$, Yun-Jian Sheng 1,2, Sarfraz Ahmed ${ }^{4}$, Changfeng Sun ${ }^{1,2}$, \\ Cun-Liang Deng ${ }^{1}$ and Suvash Chandra Ojha ${ }^{1,2 *}$

\begin{abstract}
${ }^{1}$ Department of Infectious Diseases, The Affiliated Hospital of Southwest Medical University, Luzhou, China, ${ }^{2}$ Southwest Medical University, Luzhou, China, ${ }^{3}$ Faculty of Medical Technology, Center of Data Mining and Biomedical Informatics, Narowal, Pakistan
\end{abstract} \\ Mahidol University, Bangkok, Thailand, ${ }^{4}$ Department of Basic Sciences, University of Veterinary and Animal Sciences Lahore,
}

Background: Treatment of bloodstream staphylococcal infections (BSI) necessitates the prompt initiation of appropriate antimicrobial agents and the rapid de-escalation of excessive broad-spectrum coverage to reduce the risk of mortality. We, therefore, aimed to demonstrate the diagnostic accuracy of nucleic acid amplification tests (NAAT) for the identification of methicillin-resistant S. aureus (MRSA) and methicillin-sensitive S. aureus (MSSA) in clinically suspected patients.

Methods: Until November 23, 2020, databases including PubMed, Scopus, Embase, and Web of Science were scanned for eligible studies. A bivariate random-effects model was used for meta-analysis of the 33 included studies obtained from 1606 citations, and pooled summary estimates with 95\% confidence intervals $(\mathrm{Cl})$ were generated.

Results: Twenty-three studies $(n=8,547)$ assessed NAAT accuracy for MSSA detection, while three studies $(n=479)$ evaluated MRSA detection in adults. The pooled NAAT sensitivity and specificity for MRSA in adults was higher [sensitivity: 0.83 (95\% Cl 0.59-0.96), specificity: 0.99 (95\% Cl 0.98-1.0)] as compared to MSSA [sensitivity: 0.76 (95\% Cl 0.69-0.82), specificity: 0.98 (95\% Cl 0.98-0.99)]. Similarly, eight studies $(n=4,089)$ investigating MSSA in pediatric population reported higher NAAT accuracy [sensitivity: 0.89 (95\% Cl 0.76-0.96), specificity: 0.98 (95\% Cl 0.97-0.98)] compared to adults. Among NAA tests, SeptiFast (real-time PCR, commercial) was frequently applied, and its diagnostic accuracy corresponded well to the overall summary estimates. A meta-regression and subgroup analysis of study design, sample condition, and patient selection method could not explain the heterogeneity $(P>0.05)$ in the diagnostic efficiency.

Conclusions: NAAT could be applied as the preferred initial tests for timely diagnosis and BSI management.

Keywords: bloodstream infection, NAAT accuracy, staphylococcal infections, pediatric population, meta-analysis 


\section{INTRODUCTION}

Bloodstream staphylococcal infection (BSI) is an urgent medical issue due to its rising incidence, associated poor outcome and the emergence of high rates of secondary infections such as osteomyelitis, septic arthritis, infective endocarditis and septic metastases $(1,2)$. Many surveillances worldwide recorded a rise in the incidence of BSI to varying degrees, including Thailand (27.4\%) (3), France (24\%) (4) and Brazil (40-70\%) (5), but the majority of documented cases are in adults. Pediatric incidence statistics are minimal, but infants younger than 1 year have been reported as having a higher incidence compared to adolescents (6). Especially in developed countries, methicillin-resistant $S$. aureus (MRSA) has been observed in over $60 \%$ of all isolated $S$. aureus, and associated MRSA mortality rates have reportedly been higher than methicillin-susceptible S. aureus (MSSA) (7). The burden of BSI is utterly immense in both clinical (8) and economic terms (9), with mortality rates ranging between 20 and $70 \%(10,11)$. Thus, rapid identification of the causative agents and detection of resistance markers in patients with BSI, such as the mecA gene, may provide clinically vital information to guide effective care on time, leading to improved patient outcomes.

Vancomycin, a glycopeptide antimicrobial agent, has frequently been included as empirical therapy for suspected BSI (12), along with the awareness that delays in initiating effective antimicrobial therapy may affect patient outcomes. Once identification and susceptibility of microorganisms are established, treatment can be optimized to target isolated bacteria, including discontinuation of vancomycin when MSSA is present. Though safe, this approach exposes the patient to widespectrum antibiotic overuse. Even short anti-MRSA treatment courses may alter host flora, expose to drug-induced toxicity, escalate multidrug-resistant pathogens, treatment-related side effects, and increase hospitalization costs (13). Vancomycin, the preferred antibiotic solution for MRSA infections, is also less effective than oxacillin in the treatment of MSSA infections (14). If the initial antibiotics are inadequate and changed after the diagnostic tests are available, the mortality rate does not improve significantly. Therefore, it is increasingly important to balance these two competing interests, namely the need for complete coverage while avoiding unnecessary medications.

Blood cultures currently represent the primary method for determining the etiology of the BSI (15). This traditional culturebased approach can be time-consuming, including growthbased assays, colony morphology and microdilution resistance tests. It takes about $48-72 \mathrm{~h}$ to classify the causative organism, even with a positive blood culture. A positive microbiological diagnosis in BSI can only be made in $\sim 20-30 \%$ of cases with a substantially higher false-negative rate $(16,17)$, and requires a certain amount of sample to begin the cultivation process. Nevertheless, obtaining adequate amounts of blood from neonates for culture is often difficult (18). Furthermore, samples collected after antibiotic exposure may reduce culturebased bacterial detection. As a result, the patients will likely miss the optimal chance of treatment. Therefore, if no pathogenic bacterial agent is detected, sepsis diagnosis is based solely on clinical symptoms, often coupled with an increase in essential biomarkers such as C-reactive protein or procalcitonin (19). For these reasons, there is an obvious need for a more rapid, yet precise tool that uses a limited blood volume to detect organism and guide antibiotic choices in patients with suspected BSI.

While the pathogen culture remains the gold standard, molecular amplification tests, which typically have a shorter turnaround time, can drastically reduce the critical time to initiate preventive and therapeutic strategies, including appropriate antibacterial therapy. Unlike traditional blood culture, NAAT relies on detecting bacterial DNA rather than the recovery of viable bacteria and is less affected by antibiotic preadministration. A previous study demonstrated a decrease in the length of anti-MRSA antibiotics in patients with BSI following a rapid diagnostic test (20). Several studies have assessed the importance of molecular techniques including PCR, real-time PCR, GeneXpert, LAMP, and FilmArray (21-25), but literature on the relevance of these tests to timely BSI management is widely scattered for any meaningful interpretation. Given the need to make clinical decisions among the pediatric and adult population, we have systematically reviewed and analyzed the available data to demonstrate NAAT diagnostic accuracy compared with microbiological culture.

\section{METHODS}

\section{Search Strategy}

This systemic review was carried out in compliance with the guidelines for Preferred Reporting Items for Systematic Reviews and Meta-Analyses (PRISMA) (26). A computerized search of the relevant studies without any restrictions was performed through PubMed (available since April 01, 1991), Scopus (available since April 01, 1992), Embase (available since July 01, 1992), Web of Science (available since December 01, 1999), and a reference study of the retrieved papers published until November 23, 2020. The search included a variation of the Boolean "OR" and "AND" operators with the following medical subject headings (MeSH): "Staphylococcus aureus," "S. aureus," "methicillin-resistant S. aureus," "MRSA," "Bloodstream infection," "Blood infection," "Blood culture," "Bacteremia," "Septicemia," "Sepsis," "Blood poisoning," "Nucleic acid amplification," "NAAT," "Molecular assay," "Loop-mediated isothermal amplification," "LAMP," "Polymerase chain reaction," "PCR," "Ligase chain reaction," "LCR," "Real-time PCR," “qPCR," "RT-PCR," “Amplicor," "SeptiFast," "ProbeTec," "Roche," "Gen-Probe," "FilmArray," "Cepheid," Abbott," "Sensitivity," "Specificity," and "Accuracy." No effort was made to obtain data from unpublished studies.

\section{Study Selection}

The MeSH terms were used to search through electronic databases for all relevant citations, and duplicates were carefully removed using the EndNote X9 software (Thomson Reuters, New York, NY, USA). The records obtained were initially scrutinized by reviewing titles and abstracts, and subsequent analysis excluded irrelevant studies. The full-text of potentially eligible studies for accuracy data was retrieved and carefully analyzed. The data collected by two independent researchers (K. 
Chen and A. A. Malik) were compared, and any comparative discrepancies were resolved by mutual consensus.

\section{Inclusion Criteria}

We included full-text, peer-reviewed, cross-sectional, randomized controlled, and case-control studies using NAAT to diagnose and compare BSI with a culture reference standard. For the index test, the studies explicitly provided True positive (TP), True negative (TN), False positive (FP) and False negative (FN) values or included sufficient information to derive 2 $\times 2$ contingency tables. All studies that met the standard BSI definition were eligible for inclusion, including fever, chills, palpitations, rapid breathing, gastrointestinal symptoms, confusion and shock.

\section{Exclusion Criteria}

Studies were considered for exclusion if they were conference proceedings, commentaries, reviews, meta-analysis, editorials, case reports, mechanism, and animal experimentation. Studies providing insufficient data for constructing a $2 \times 2$ contingency table and comprising $<10$ specimens were excluded. Noninterpretable test results by both index test and microbiological reference standard were also not included.

\section{Data Extraction}

Two independent analysts (K. Chen and A. A. Malik) scanned all the related papers with pre-specified eligibility requirements in order to ensure the reproducibility of study selection. Disagreed studies were resolved by consultation with a third investigator (S. C. Ojha). The data were collected from eligible studies including authorship, publishing year, country, settings, study type, patient selection, patient characteristics, sample type, sample size, NAAT specifics, potential risks, and information for construction of 2 $\times 2$ contingency table. For missing details, ambiguous reference standards, and specimen preparation techniques, the authors were consulted individually. Contingency tables for NAAT performance compared to microbiological reference standards were constructed on the basis of available data from the qualifying studies. Studies involving different index tests as compared to the specific reference standard were considered separate studies.

\section{Quality Assessment}

The methodological quality of the studies was assessed using the Quality Assessment of Diagnostic Accuracy Studies (QUADAS2), a validated diagnostic study tool (27). The four QUADAS-2 domains were: patient selection, index test, reference standard, and flow and timing. All four domains for the possible risk of bias and the first three domains for applicability concerns were evaluated by reviewing authors (K. Chen and A. A. Malik). Each domain was assessed in terms of risk of bias using signaling questions that are answered with "yes," "no," or "unclear" and are judged as "low," "high," or "unclear," respectively. The first three domains are simultaneously evaluated in terms of applicability concerns, which are also graded as "low," "high," or "unclear" with identical characteristics. The differences between the reviewing authors were settled by consensus.

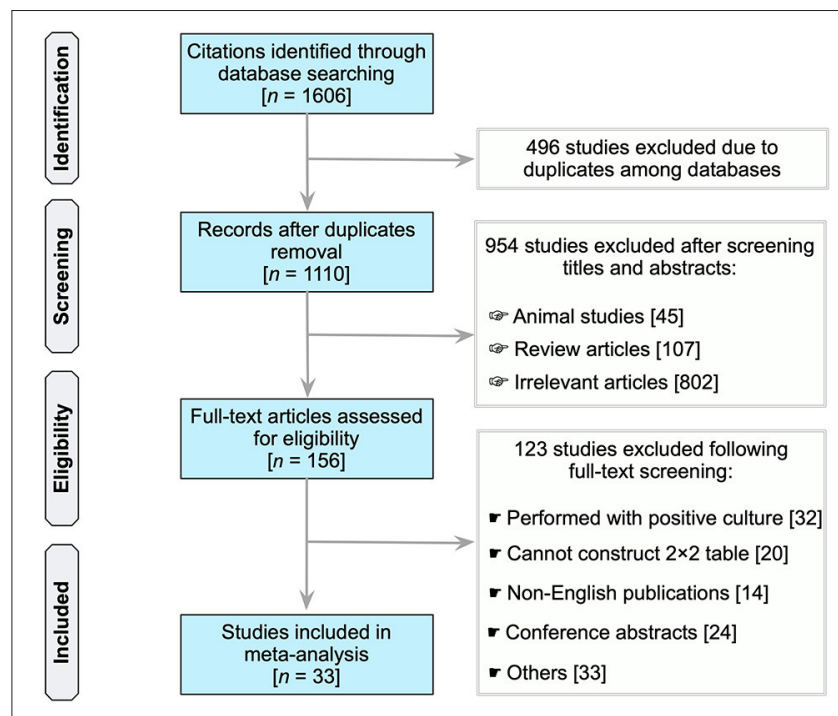

FIGURE 1 | Flow chart of study selection.

\section{Statistical Analysis}

The following software was used for data analysis: RevMan 5.4 (Nordic Cochrane Centre, Copenhagen, Denmark) for the methodological quality assessment of included studies and summary plots generation (28). Meta-DiSc 1.4 (Cochrane Colloquium, Barcelona, Spain) for computation of pooled summary estimates including specificity, sensitivity, likelihood ratios, diagnostic odds ratio (DOR), and heterogeneity amongst data (29). Diagnostic accuracy of NAAT in association with 95\% CI was computed against microbiological culture using a random-effects model. Furthermore, the $I$-square $\left(I^{2}\right)$ statistics were used to evaluate the heterogeneity of the included studies, where $I^{2}$ values ranging from 0 to $40 \%$ indicate low heterogeneity, 30-60\% moderate heterogeneity, $50-90 \%$ substantial heterogeneity, and values $>90 \%$ signify considerable heterogeneity (30). Using subgroup analysis, different specimen conditions, patient selection, study design, and patient type were analyzed as possible heterogeneity sources. Publication bias was inspected using Deeks' funnel plot asymmetry test (31). A $P$-value of $<0.05$ was generally considered to be statistically significant.

\section{RESULTS}

\section{Literature Selection}

In total, our search identified 1,606 unique records (PubMed, 402; Scopus, 818; Embase, 180; Web of Science, 206) (Figure 1). Of which, due to duplication in databases, 496 citations were eliminated. Following the scanning of the titles and abstracts of 1,110 publications, 156 studies considered potentially significant were subjected to a full-text revision. Supplementary Table 1 summarizes the studies reviewed, includin why these studies were excluded (see Supplementary Table 1). Essentially, 33 studies were included in subsequent analyses that met all the inclusion criteria (21, 32-63). 
TABLE 1 | Baseline features of included studies.

\begin{tabular}{|c|c|c|c|c|c|c|c|c|c|c|}
\hline References & Year & Country & Setting & $\begin{array}{l}\text { Pros } \\
\text { enroll }\end{array}$ & Patients selection & Patient type & Sample type & Sample size & NAAT specifics & Potential risks \\
\hline Abd El-Aziz et al. (32) & 2020 & Egypt & TCC & No & Convenience & Pediatric & Fresh & 30 & qPCR & Sepsis \\
\hline Arabestani et al. (33) & 2014 & Iran & UHL & No & Convenience & Adult & Fresh/Frozen & 126 & mPCR & Bacteremia \\
\hline Bloos et al. (34) & 2010 & Germany & TCC & Yes & Convenience & Adult & Fresh/Frozen & 347 & SeptiFast & Sepsis \\
\hline Etchebarne et al. (35) & 2017 & USA & TCC & Yes & Consecutive & Adult & Fresh & 31 & LAMP & Sepsis \\
\hline Faraji et al. (36) & 2018 & Iran & UHL & No & Convenience & Adult & Fresh & 20 & qPCR & $\mathrm{IE}$ \\
\hline Fernández-Romero et al. (37) & 2014 & Spain & UHL & Yes & Convenience & Adult & Fresh & 96 & SeptiFast & BSI, SIRS \\
\hline García-Gudiño et al. (38) & 2018 & Mexico & TCC & No & Convenience & Pediatric & Fresh/Frozen & 22 & PCR-DGGE & Sepsis \\
\hline Ginn et al. (39) & 2017 & Australia & TCC & No & Convenience & Adult & Fresh/Frozen & 15 & MT-PCR & Sepsis \\
\hline Grosse-Onnebrink et al. (40) & 2017 & Germany & UHL & Yes & Convenience & Adult & Fresh & 72 & SeptiFast & CF \\
\hline Josefson et al. (41) & 2011 & Sweden & UHL & Yes & Consecutive & Adult & Fresh/Frozen & 1540 & SeptiFast & $\mathrm{BSI}$ \\
\hline Kitagawa et al. (42) & 1996 & Japan & UHL & No & Convenience & Adult & Fresh & 41 & Nested PCR & Bacteremia \\
\hline Knabl et al. (43) & 2016 & Austria & UHL & Yes & Consecutive & Adult & Fresh & 58 & SeptiFast & SIRS \\
\hline Korber et al. (44) & 2017 & Austria & TCC & No & Convenience & Adult & Fresh & 470 & SeptiFast & Sepsis \\
\hline Lehmann et al. (45) & 2009 & Germany & UHL & No & Convenience & Adult & Fresh & 467 & SeptiFast & Sepsis \\
\hline Liberto et al. (46) & 2006 & Italy & TCC & No & Convenience & Adult & Fresh & 31 & qPCR-M & Bacteremia \\
\hline Liu et al. (47) & 2017 & China & TCC & No & Convenience & Adult & Fresh & 30 & qPCR & Sepsis \\
\hline Lucignano et al. (48) & 2011 & Italy & TCC & No & Convenience & Pediatric & Fresh & 1,673 & SeptiFast & Sepsis \\
\hline Makhoul et al. (21) & 2005 & Israel & TCC & Yes & Convenience & Pediatric & Fresh & 215 & PCR & Bacteremia \\
\hline Moore et al. (49) & 2018 & Uganda & TCC & No & Convenience & Adult & Fresh/Frozen & 336 & qPCR-TAC & Sepsis \\
\hline Obara et al. (50) & 2011 & Japan & UHL & Yes & Convenience & Adult & Fresh & 78 & SeptiFast & Bacteremia \\
\hline Oeser et al. (51) & 2020 & UK & TCC & No & Convenience & Pediatric & Fresh/Frozen & 208 & qPCR & Sepsis \\
\hline Pasqualini et al. (52) & 2012 & Italy & UHL & Yes & Consecutive & Adult & Fresh & 391 & SeptiFast & SIRS \\
\hline Peters et al. (53) & 2007 & Netherlands & UHL & No & Convenience & Adult & Fresh/Frozen & 175 & qPCR & Bacteremia \\
\hline Rogina et al. (54) & 2014 & Slovenia & TCC & No & Consecutive & Adult & Fresh & 23 & SeptiTest, IHP & SIRS, Sepsis \\
\hline Santolaya et al. (55) & 2011 & Chile & TCC & Yes & Convenience & Pediatric & Fresh/Frozen & 177 & RT-PCR & Bacteremia \\
\hline Schaub et al. (56) & 2014 & Switzerland & UHL & Yes & Convenience & Adult & Fresh/Frozen & 110 & SeptiFast & SIRS, Sepsis \\
\hline Van den Brand et al. (57) & 2018 & Netherland & UHL & Yes & Convenience & Pediatric & Fresh/Frozen & 91 & mPCR & Sepsis \\
\hline Wallet et al. (58) & 2010 & France & TCC & Yes & Consecutive & Adult & Fresh & 100 & SeptiFast & Sepsis \\
\hline Wu et al. (59) & 2011 & Italy & TCC & No & Convenience & Pediatric & Fresh & 1,673 & mPCR & Sepsis \\
\hline Xiao et al. (60) & 2019 & China & UHL & No & Consecutive & Adult & Fresh/Frozen & 2,844 & PCR-MCA & BSI \\
\hline Yanagihara et al. (61) & 2010 & Japan & UHL & Yes & Convenience & Adult & Fresh/Frozen & 407 & SeptiFast & SIRS \\
\hline Zboromyrska et al. (62) & 2016 & Spain & TCC & Yes & Convenience & Adult & Fresh & 92 & GeneXpert & CRB \\
\hline Ziegler et al. (63) & 2016 & Sweden & UHL & No & Consecutive & Adult & Fresh/Frozen & 696 & MST & Sepsis \\
\hline
\end{tabular}

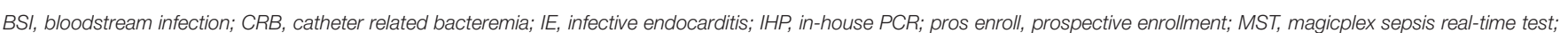

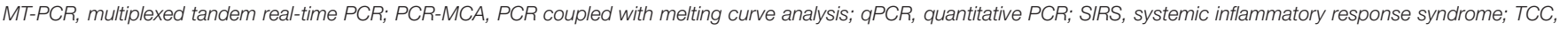
tertiary care center (hospital); UHL, university hospital laboratory.

\section{Characteristics of the Included Studies}

Table 1 shows the baseline features of the studies included (21, 32-63). Twenty-six studies were performed in countries with high incomes and seven in countries with lower-middle incomes. Studies reporting the usefulness of multiple index tests against a single reference standard were treated as separate studies. Centered upon this theory, this meta-analysis included 33 publications comprising 35 datasets. Out of 23 studies, 24 datasets $(n=8,547)$ evaluated the accuracy of NAAT for MSSA detection, while three studies $(n=479)$ examined the NAAT accuracy for MRSA detection in adults. Similarly, eight studies ( $n$ $=4,089$ ) evaluated NAAT's accuracy for the identification of BSI in children. The total number of samples submitted for diagnostic assessment ranged from 15 to 2,844, with a median value of 100 .
All experimental tests were carried out in tertiary care hospitals or university research facilities. Only studies published in English before November 23, 2020, were included.

\section{Quality Appraisal}

We applied the QUADAS-2 tool to evaluate the methodological quality of included studies against microbiological culture reference standard (see Figure 2). For a few studies in the patient selection domain $(34,38,40,48,53,55,63)$, the risk of bias was high because the studies could not prevent improper sample exclusion. Regarding the applicability concern, all studies included blood samples from patients suspected of BSI, indicating a low risk of bias. Other domains, including the index test and the reference standard, were presumably at 


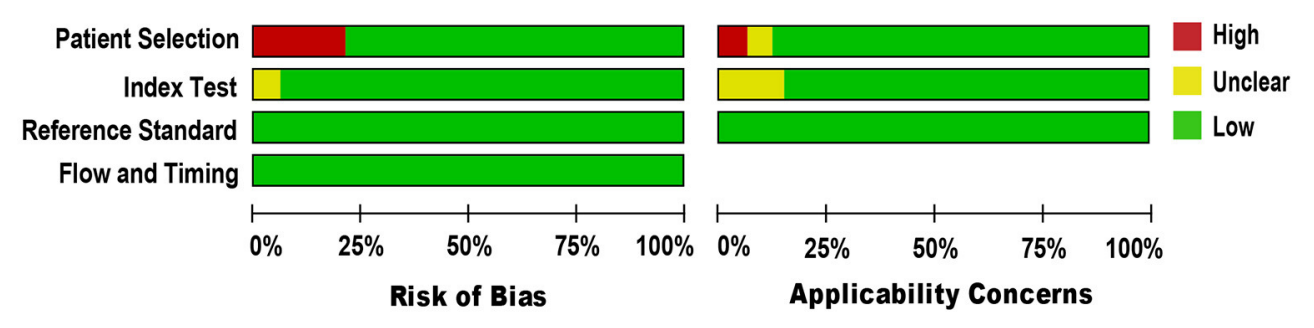

FIGURE 2 | Methodological quality and risk of bias assessment of the eligible studies.

$\begin{array}{lrrrrrr}\text { Study } & \text { TP } & \text { FP } & \text { FN } & \text { TN } & \text { Sensitivity }(95 \% \text { CI) } & \text { Specificity }(95 \% \text { Cl) } \\ \text { Abd El-Aziz [32] } & 0 & 4 & 0 & 26 & \text { Not estimable } & 0.87[0.69,0.96] \\ \text { Garcia-Gudiño [38] } & 0 & 2 & 1 & 19 & 0.00[0.00,0.97] & 0.90[0.70,0.99] \\ \text { Lucignano [48] } & 23 & 8 & 0 & 0 & 1.00[0.85,1.00] & 0.00[0.00,0.37] \\ \text { Makhoul [21] } & 4 & 0 & 0 & 211 & 1.00[0.40,1.00] & 1.00[0.98,1.00] \\ \text { Oeser [51] } & 0 & 28 & 0 & 180 & \text { Not estimable } & 0.87[0.81,0.91] \\ \text { Santolaya [55] } & 3 & 17 & 0 & 157 & 1.00[0.29,1.00] & 0.90[0.85,0.94] \\ \text { van den Brand [57] } & 8 & 3 & 3 & 77 & 0.73[0.39,0.94] & 0.96[0.89,0.99] \\ \text { Wu [59] } & 23 & 8 & 0 & 1642 & 1.00[0.85,1.00] & 1.00[0.99,1.00]\end{array}$

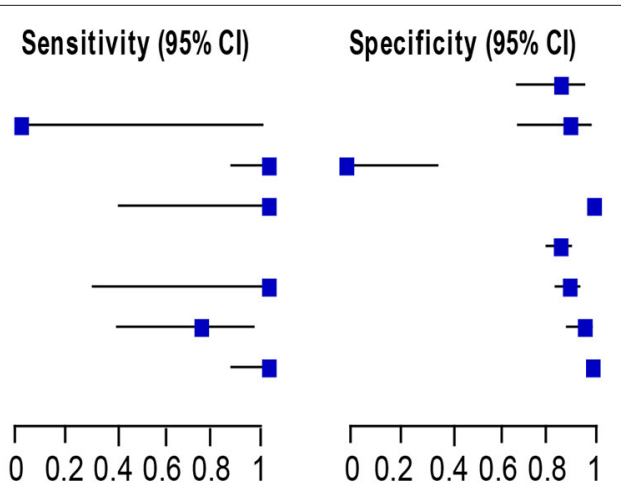

FIGURE 3 | Forest plot for detection of MSSA in the pediatric population. The square stands for the sensitivity and specificity of a particular study, the black line represents its confidence interval. TP, true positive, FP, false positive, $\mathrm{FN}$, false negative, TN, true negative, $\mathrm{Cl}$, confidence interval.

low risk of bias, as NAAT used pre-established binary response investigation criteria. Concerns about the index test conduct's applicability are unclear, as no proven protocol is available for global use. Reference standards of all studies have been carried out in hospitals or university-affiliated reference labs; as such, we expect operator error bias to be of low concern. Subsequently, there was no uncertainty regarding the possible risk of bias in the flow and timing domain as both the index test and the reference standards were applied to the same samples. In general, the quality of the studies included in our meta-analysis met the methodological standards.

\section{Summary Estimates}

The studies were rather heterogeneous, so obtaining NAAT pooled summary estimates from integrated pediatric and adult populations was not considered meaningful for antimicrobial therapy. Initially, we concentrated on diagnosing BSI in children as it is often difficult to procure sufficiently large amounts of blood from children for microbiological culture. Subsequently, the pooled summary estimates of NAAT in the adult population to diagnose BSI were assessed. The accuracy of index tests, commercial tests, and a potential source of heterogeneity among studies in predefined subgroups have also been demonstrated.

\section{Detection of BSI Among the Pediatric Population}

Eight studies $(21,32,38,48,51,55,57,59)$ met the inclusion criteria for comparing NAAT with a microbiological culture among children consisting of a total of 4,089 samples for MSSA detection in suspected BSI patients. The sensitivity and specificity of NAAT for MSSA detection ranged from 0.25 (95\% CI $0.0-$ 0.94 ) to 1.0 (95\% CI, 0.85-1.0), whilst our search did not result in any MRSA studies (Figure 3). The pooled summary estimates of NAAT for detection of MSSA in blood were [sensitivity: 0.89 (95\% CI 0.76-0.96), specificity: 0.98 (95\% CI 0.97-0.98), positive likelihood ratio (PLR): 26.9 (95\% CI 6.35-114.2), negative likelihood ratio (NLR): 0.25 (95\% CI 0.08-0.8), DOR: 142.34 (95\% CI 16.85-1,202.7)]. The $I^{2}$ sensitivity and specificity statistical values were 57.0 and $95.7 \%$, respectively, indicating substantial to considerable heterogeneity. The area under the curve (AUC) of summary receiver operating characteristics (SROC) was 0.90 (95\% CI 0.74-1.0), indicating overall justifiable diagnostic validity (Figure 5A).

\section{Detection of BSI Among the Adult Population}

Of 23 studies (33-37, 39-47, 49, 50, 52-54, 56, 58, 60-63), 24 datasets consisting of 8,547 blood samples evaluated the accuracy of NAAT for MSSA identification, whereas three datasets ( $n$ $=479$ ) assessed the NAAT accuracy for MRSA identification. The sensitivity and specificity of NAAT for identification of MSSA ranged from 0.17 (95\% CI $0.0-0.82)$ to 1.0 (95\% CI $0.85-1.00)$ and from 0.90 (95\% CI $0.73-0.98)$ to 1.00 (95\% CI $0.99-1.00$ ), respectively (Figure 4A). While the sensitivity and specificity of NAAT for identification of MRSA ranged from 0.25 (95\% CI $0.0-0.94)$ to 1.0 (95\% CI 0.74-1.00) and from 
A

$\begin{array}{lrrrrr}\text { Study } & \text { TP } & \text { FP } & \text { FN } & \text { TN } & \text { Sensitivity }(95 \% \text { Cl } \\ \text { Arabestani [33] } & 4 & 3 & 1 & 118 & 0.80[0.28,0.99] \\ \text { Bloos [34] } & 7 & 21 & 8 & 311 & 0.47[0.21,0.73] \\ \text { Faraji [36] } & 0 & 1 & 0 & 19 & \text { Not estimable } \\ \text { Fernández-Romero [37] } & 4 & 2 & 1 & 89 & 0.80[0.28,0.99] \\ \text { Ginn [39] } & 0 & 0 & 2 & 13 & 0.00[0.00,0.84] \\ \text { Grosse-Onnebrink [40] } & 0 & 0 & 1 & 71 & 0.00[0.00,0.97] \\ \text { Josefson [41] } & 10 & 13 & 5 & 1512 & 0.67[0.38,0.88] \\ \text { Knabl [43] } & 0 & 0 & 1 & 57 & 0.00[0.00,0.97] \\ \text { Korber [44] } & 9 & 8 & 1 & 452 & 0.90[0.55,1.00] \\ \text { Lehmann [45] } & 13 & 16 & 1 & 437 & 0.93[0.66,1.00] \\ \text { Liberto [46] } & 9 & 0 & 0 & 22 & 1.00[0.66,1.00] \\ \text { Liu [47] } & 1 & 3 & 0 & 26 & 1.00[0.03,1.00] \\ \text { Moore [49] } & 1 & 3 & 3 & 329 & 0.25[0.01,0.81] \\ \text { Obara [50] } & 2 & 2 & 0 & 74 & 1.00[0.16,1.00] \\ \text { Pasqualini [52] } & 8 & 3 & 3 & 377 & 0.73[0.39,0.94] \\ \text { Peters [53] } & 21 & 10 & 7 & 125 & 0.75[0.55,0.89] \\ \text { Rogina [54] } & 2 & 0 & 0 & 21 & 1.00[0.16,1.00] \\ \text { Rogina [54] } & 0 & 0 & 2 & 21 & 0.00[0.00,0.84] \\ \text { Schaub [56] } & 3 & 1 & 1 & 74 & 0.75[0.19,0.99] \\ \text { Wallet [58] } & 1 & 4 & 0 & 96 & 1.00[0.03,1.00] \\ \text { Xiao [60] } & 22 & 0 & 0 & 2822 & 1.00[0.85,1.00] \\ \text { Yanagihara [61] } & 3 & 3 & 0 & 401 & 1.00[0.29,1.00] \\ \text { Zboromyrska [62] } & 4 & 3 & 0 & 85 & 1.00[0.40,1.00] \\ \text { Ziegler [63] } & 14 & 35 & 5 & 642 & 0.74[0.49,0.91]\end{array}$

Specificity $(95 \% \mathrm{Cl})$

$0.98[0.93,0.99]$

$0.94[0.90,0.96]$

$0.95[0.75,1.00]$

$0.98[0.92,1.00]$

$1.00[0.75,1.00]$

$1.00[0.95,1.00]$

$0.99[0.99,1.00]$

$1.00[0.94,1.00]$

$0.98[0.97,0.99]$

$0.96[0.94,0.98]$

$1.00[0.85,1.00]$

$0.90[0.73,0.98]$

$0.99[0.97,1.00]$

$0.97[0.91,1.00]$

$0.99[0.98,1.00]$

$0.93[0.87,0.96]$

$1.00[0.84,1.00]$

$1.00[0.84,1.00]$

$0.99[0.93,1.00]$

$0.96[0.90,0.99]$

$1.00[1.00,1.00]$

$0.99[0.98,1.00]$

$0.97[0.90,0.99]$

$0.95[0.93,0.96]$

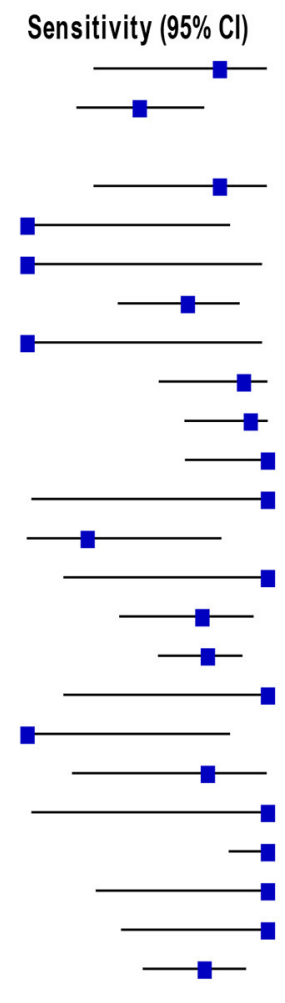

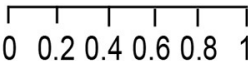

Specificity $(95 \% \mathrm{Cl})$

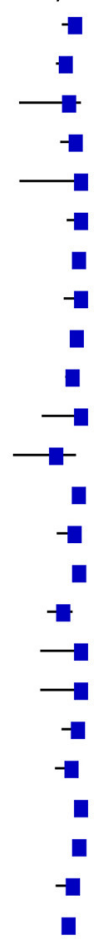

00.20 .40 .60 .81

B

\begin{tabular}{|c|c|c|c|c|c|c|c|}
\hline Study & TP & FP & FN TN & Sensitivity $(95 \% \mathrm{Cl})$ & Specificity $(95 \%$ Cl) & Sensitivity (95\% Cl) & Specificity $(95 \%$ Cl) \\
\hline Etchebarne [35] & 0 & 0 & 130 & $0.00[0.00,0.97]$ & $1.00[0.88,1.00]$ & 0 & $\rightarrow$ \\
\hline Kitagawa [42] & 12 & 2 & $\begin{array}{ll}0 & 27\end{array}$ & $1.00[0.74,1.00]$ & $0.93[0.77,0.99]$ & $\longrightarrow$ & \\
\hline Yanagihara [61] & 4 & 0 & 2401 & $0.67[0.22,0.96]$ & $1.00[0.99,1.00]$ & $\longrightarrow$ & ! \\
\hline
\end{tabular}

FIGURE 4 | Forest plot for detection of (A) MSSA and (B) MRSA in the adult population. The square stands for the sensitivity and specificity of a particular study, the black line represents its confidence interval. TP, true positive, FP, false positive, FN, false negative, TN, true negative, $\mathrm{Cl}$, confidence interval.

0.93 (95\% CI $0.77-0.99)$ to 1.00 (95\% CI 0.99-1.0), respectively (Figure 4B). Pooled summary estimates of NAAT for MSSA identification was lower [sensitivity: 0.76 (95\% CI $0.69-0.82)$, specificity: 0.98 (95\% CI 0.98-0.99), PLR: 28.63 (95\% CI $18.59-$ 44.1), NLR: 0.34 (95\% CI 0.23-0.50), DOR: 116.38 (95\% CI 57.68-234.8)] compared to MRSA [sensitivity: 0.83 (95\% CI 0.59-0.96), specificity: 0.99 (95\% CI 0.98-1.0), PLR: 40.73 (95\% CI 3.89-426.1), NLR: 0.32 (95\% CI 0.08-1.32), DOR: 268.6 (95\% CI 32.1-2250.0)]. The $I^{2}$ statistical scores for sensitivity and specificity of MSSA identification were 57.4 and $88.9 \%$, respectively, indicating substantial to considerable heterogeneity. While $I^{2}$ statistical scores for sensitivity and specificity of MRSA were 76.9 and $82.1 \%$, indicating considerable heterogeneity. The
AUC of SROC for assorted MSSA and MRSA among adult population was 0.97 (95\% CI 0.94-1.0), suggesting overall valid diagnostic accuracy (Figure 5B).

\section{Diagnostic Accuracy of In-house vs. Commercial Tests}

The diagnostic accuracy of studies based on various NAA tests is summarized in Table 2. For pediatric and adult populations, the pooled summary estimates of the in-house NAA tests for detecting MSSA were consistently higher (sensitivity $\geq 78 \%$; specificity $\geq 93 \%$ ). The pooled summary estimates of the commercial tests were higher for MSSA identification [sensitivity: 0.75 (95\% CI $0.66-0.82$ ), specificity: 0.98 (95\% CI $0.97-0.98$ ), 


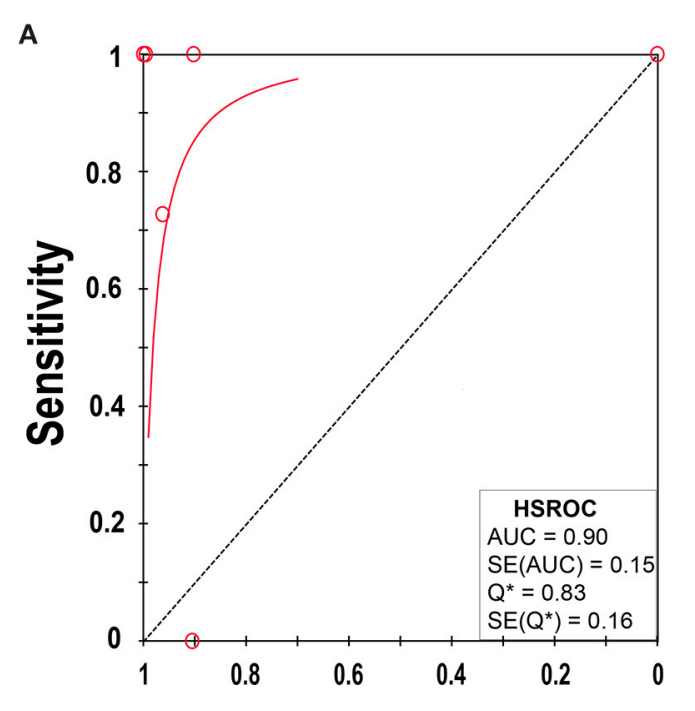

B

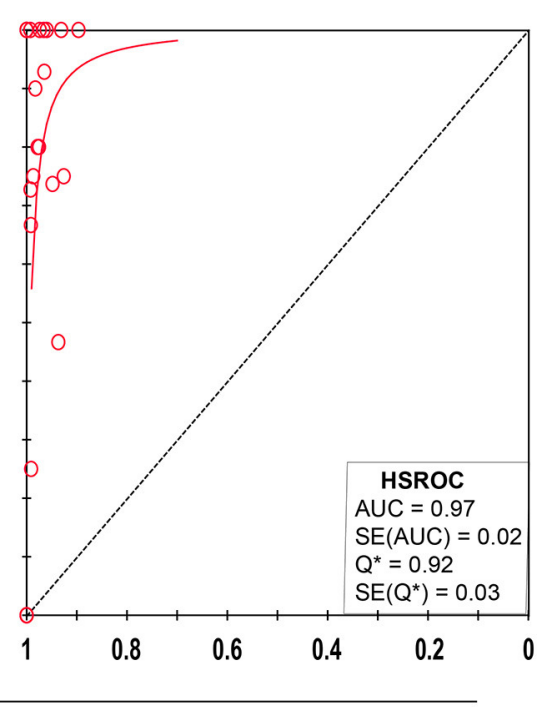

\section{Specificity}

FIGURE 5 | SROC plot of NAAT for (A) pediatric and (B) adult population. Red circles indicate the data point from each of the investigations, and the solid blue line represents the SROC curve.

PLR: 31.9 (95\% CI 20.4-50.0), NLR: 0.3 (95\% CI $0.2-0.5$ ), DOR: 126.1 (95\% CI 62.1-256.2) and AUC 0.98 (95\% CI 0.960.99)] as compared to MRSA [sensitivity: 0.56 (95\% CI $0.21-$ 0.88), specificity: 1.0 (95\% CI 0.99-1.0), PLR: 113.2 (95\% CI 4.7-2,699.8), NLR: 0.53 (95\% CI 0.26-1.0), and DOR: 234.2 (95\% CI 4.65-11,801.6)]. Among NAAT studies, SeptiFast were consistently performed to detect BSI in blood samples compared to other tests.

\section{Meta-Regression and Subgroup Analysis}

We assessed the possible source of heterogeneity through a meta-regression analysis on predefined subgroups. Metaregression suggested that study design (prospective/others), country (developed/developing), and patient selection (consecutive/convenience) were not the significant source of heterogeneity (meta-regression $P=0.42, P=0.34$, and $P=$ 0.62 , respectively) with the exception of the sample condition (fresh/frozen) $(P=0.04)$.

\section{Publication Bias}

Using Deek's funnel plot asymmetry test, publication bias was measured. In our analysis, we did not find striking publication bias $(P=0.25)$.

\section{DISCUSSION}

Despite advances in supportive care, BSI is a leading cause of death worldwide (10). In recent years, most studies have centered on screening positive blood culture to identify causative staphylococcal pathogen (64-66); however, it remains a matter of concern as every hour of delay in initiating effective antimicrobial therapy increases mortality by $7.6 \%$ in sepsis patients (67).
Therefore, it is essential to recognize staphylococcal species and their resistance markers rapidly in patients with suspected BSI, as the prompt intervention will lead to improved clinical outcomes with effective antimicrobial therapy. Currently, several multiplex molecular assays have been cleared by the Food and Drug Administration (FDA) that can identify a wide range of microorganisms concurrently with specific resistance genes in blood samples, and several of these assays have been rendered commercially accessible (68). Nevertheless, the numerous case descriptions and the diverse samples used in the various studies make it difficult to compare research results and restrict diseases' management. We, therefore, conducted a systematic review and meta-analysis to evaluate NAAT's diagnostic performance for diagnosing BSI in clinically suspected patients.

Our results revealed that NAAT overall summary estimates for MRSA detection [sensitivity: 0.83 (95\% CI 0.59-0.96), specificity: 0.99 (95\% CI 0.98-1.0), AUC: 0.98 (95\% CI 0.96-0.99)] were higher as compared to MSSA [sensitivity: 0.76 (95\% CI 0.690.82), specificity: 0.98 (95\% CI 0.98-0.99), AUC: 0.98 (95\% CI 0.97-0.99)] among adults. Relatively smaller sample size, different DNA extraction technique, target genes adopted, and reaction material quality are among factors that could have contributed to greater NAAT accuracy for MRSA detection. Figure 6 shows the pooled sensitivity and specificity of NAAT for BSI detection in children and adults.

In the pediatric population, NAAT displayed consistently higher diagnostic accuracy for MSSA detection [sensitivity: 0.89 (95\% CI 0.76-0.96), specificity: 0.98 (95\% CI 0.97-0.98), AUC: 0.90 (95\% CI 0.74-1.0)]. Albeit not perfect, the higher sensitivity of NAAT among a pediatric population, where the acquisition of large volumes of blood is a major concern, and relatively small non-interpretable results, encourages the use of the test for 
TABLE 2 | Subgroup analysis of studies based on different NAA tests.

\begin{tabular}{|c|c|c|c|c|c|c|c|c|c|c|}
\hline Subject & Diagnostic target & Subgroup & Total data & NAAT methods & \% Sensitivity $(95 \% \mathrm{Cl})$ & \% Specificity (95\% Cl) & PLR (95\% CI) & NLR (95\% Cl) & DOR $(95 \% \mathrm{Cl})$ & AUC $(95 \% \mathrm{Cl})$ \\
\hline \multirow[t]{14}{*}{ Adult } & S. aureus & In-house & 8 & & $79(66-88)$ & $100(99-100)$ & $23.4(6.5-84.7)$ & $0.3(0.1-0.9)$ & $91.7(12.3-684.4)$ & 86 (84-88) \\
\hline & & & & qPCR (3) & 75 (56-89) & $92(88-96)$ & $9.3(5.3-16.2)$ & $0.3(0.2-0.5)$ & $34.7(12.8-93.8)$ & $94(92-97)$ \\
\hline & & & & PCR (2) & 56 (20-88) & $98(94-100)$ & $28.3(9.2-87.2)$ & $0.4(0.1-3.0)$ & $67.6(6.75-677.9)$ & - \\
\hline & & & & PCR-MCA (1) & $100(85-100)$ & $100(100-100)$ & - & 0.0 & - & $100(99-100)$ \\
\hline & & & & MT-PCR (1) & $0(0-84)$ & $100(75-100)$ & - & $1.0(1.0-1.0)$ & - & 87 (60-98) \\
\hline & & Commercial & 17 & & $75(66-82)$ & $98(97-98)$ & $31.9(20.4-50.0)$ & $0.3(0.2-0.5)$ & $126.1(62.1-256.2)$ & 98 (96-99) \\
\hline & & & & SeptiFast (14) & $76(66-84)$ & 98 (98-99) & $36.9(21.9-62.4)$ & $0.3(0.2-0.5)$ & $160.8(66.8-387.2)$ & $99(97-100)$ \\
\hline & & & & qPCR TAC (1) & $25(63-81)$ & $99(97-100)$ & $27.7(3.6-212.2)$ & $0.8(0.4-1.3)$ & - & 98 (96-99) \\
\hline & & & & GeneXpert (1) & $100(40-100)$ & 97 (90-99) & $29(9.7-89.2)$ & 0.0 & - & 97 (91-99) \\
\hline & & & & MST (1) & 74 (49-91) & 95 (93-96) & $14.3(9.4-21.7)$ & $0.3(0.1-0.6)$ & - & $94(92-96)$ \\
\hline & MRSA & In-house & 1 & Nested PCR (1) & $100(74-100)$ & 93 (77-99) & $14.5(3.8-55.2)$ & 0.0 & - & 95 (84-99) \\
\hline & & Commercial & 2 & & $56(20-88)$ & $100(99-100)$ & $113(4.7-2699.8)$ & $0.53(0.26-1.0)$ & $234(4.65-11801.6)$ & - \\
\hline & & & & LAMP (1) & 0 (0-98) & $100(88-100)$ & - & $1.0(1.0-1.0)$ & - & $97(83-100)$ \\
\hline & & & & SeptiFast (1) & 67 (22-96) & $100(99-100)$ & - & $0.3(0.1-1.0)$ & - & $100(98-100)$ \\
\hline \multirow[t]{6}{*}{ Children } & S. aureus & In-house & 7 & & 78 (57-92) & 96 (95-97) & $17.5(5.0-61.8)$ & $0.4(0.2-0.8)$ & $72.2(9.9-528.0)$ & $74(57-97)$ \\
\hline & & & & qPCR (4) & 75 (48-93) & 89 (86-92) & $9.1(4.8-17.4)$ & $0.3(0.2-0.7)$ & $39.7(10.4-151.2)$ & $93(83-100)$ \\
\hline & & & & GSPBRT-PCR (1) & $100(29-100)$ & $100(99-100)$ & - & 0.0 & - & $100(99-100)$ \\
\hline & & & & PCR-DGGE (1) & $0(0-98)$ & 91 (70-99) & 0.0 & $1(0.9-1.3)$ & - & $86(65-97)$ \\
\hline & & & & PCR (1) & $100(40-100)$ & $100(98-100)$ & - & 0.0 & - & - \\
\hline & & Commercial & 1 & SeptiFast (1) & $100(85-100)$ & $100(99-100)$ & 206 (103-412) & 0.0 & - & $100(99-100)$ \\
\hline
\end{tabular}

not estimable; AUC, area under curve; DOR, diagnostic odds ratio; LAMP, loop-mediated isothermal amplification; NAAT, nucleic acid amplification tests; NLR, negative likelihood ratio; PLR, positive likelihood ratio; qPCR, quantitative PCR. 


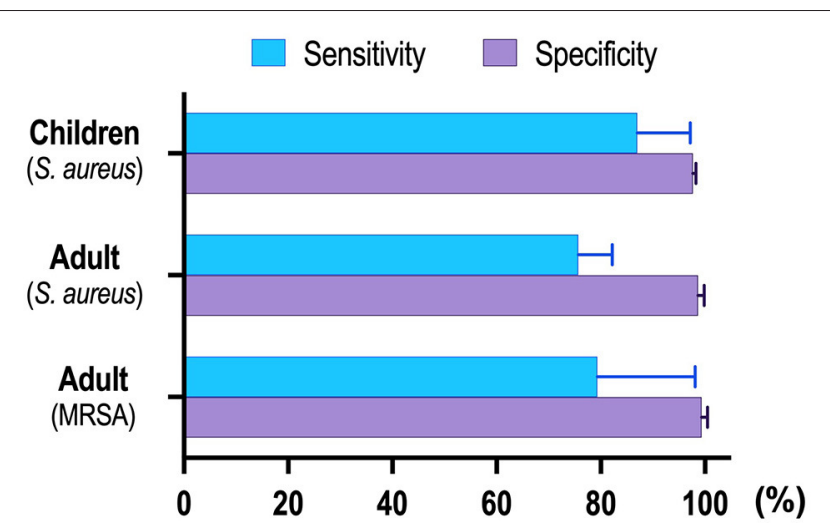

FIGURE 6 | Summary for NAAT's pooled sensitivity and specificity.

the diagnosis of BSI in principle. Furthermore, NAAT identified more pathogens than blood cultures alone in all studies, and specificity ( $\sim 98 \%)$ of the test was consistent among pediatric and adult populations, highlighting higher NAAT diagnostic accuracy. Compared to previously published systematic reviews conducted by Pammi et al. (69) in the neonatal population, we found that the pooled sensitivity of 0.90 (95\% CI 0.82-0.95) and specificity of 0.93 (95\% CI $0.89-0.96)$ were similar to our study. Similarly, a meta-analysis by Su et al. (70) mainly summarized the diagnostic value of the 16S rRNA gene PCR test for neonates, which reported a nearly similar sensitivity of 0.85 (95\% CI, $0.81-0.88)$ and a specificity of 0.96 (95\% CI, 0.95-0.96) for BSI diagnosis. All these reviews were not unique to MSSA or MRSA for the definitive diagnosis of BSI.

Additionally, NAAT subgroup analysis found that in-house tests [sensitivity: 0.78 (95\% CI 0.66-0.88), specificity: 0.99 (95\% CI 0.99-1.0)] and commercial tests [sensitivity: 0.75 (95\% CI 0.66-0.82), specificity: 0.98 (95\% CI 0.97-0.98)] were comparable for MSSA detection (Table 2). The PLR for commercial research was 31.9, implying that patients with BSI are $\sim 32$ times more likely than patients without BSI to be NAA test positive. In the case of MRSA, there was insufficient data in the inhouse and commercial test subgroups to allow meaningful comparisons. Searching for more accurate commercial test details, we observed that SeptiFast was often used in BSI diagnosis, and the pooled summary estimates of tests [sensitivity: 0.76 (95\% CI 0.66-0.84), specificity: 0.98 (95\% CI 0.98-0.99)] corresponded well-with the study by Chang and colleagues [sensitivity: 0.75 (95\% CI 0.65-0.83), specificity: 0.92 (95\% CI 0.90-0.95)] (71). However, Chang and colleagues evaluated the accuracy of SeptiFast against a composite reference standard, including bacteremia and fungemia, which was not unique to BSI.

The vital strengths of this study include a rigorous search strategy, utility of systemic guidelines, impartial selection criteria, a precise reference standard, a bivariate random-effects model for data-manipulation, meta-regression analysis on predefined subgroups, and independent analysts' interpretation. Studies that did not adhere to specific guidelines for diagnosing BSI, as well as those with a high risk of bias and high applicability concerns, as judged by the QUADAS-2 tool, were removed from subsequent analysis. We also excluded studies with fewer than ten samples to reduce the effects of publication bias while also discouraging future researchers from conducting small-scale studies, which is consistent with other meta-analyses $(72,73)$. Furthermore, and involved pre-enrichment steps before molecular testing, which may tend to overstate the index test's diagnostic performance, were excluded.

There are a few limitations to our analysis. It is likely that we may have overlooked a few crucial studies through systematic literature searches across databases. Due to the high degree of reporting variability of the included studies, the effect of factors such as sample volume, non-standardized sample preparation, NAA testing expertise, amplification procedures, and laboratory facilities on the accuracy of NAA tests could not be addressed. It should be noted that the gene targets were also different, which could be possible reasons for the heterogeneity. Although the study design, sample condition, and patient selection were not significant sources of heterogeneity in the meta-regression analysis, these variables could enhance heterogeneity and limit the generalizability of the NAAT's overall diagnostic accuracy. In addition, this meta-analysis was constrained due to insufficient MRSA studies among both children and adult populations, and should be interpreted with caution.

\section{CONCLUSIONS}

Our findings suggest that currently available molecular assays may not have adequate diagnostic accuracy to replace microbial cultures. However, molecular assays have a shorter turnaround time, a higher proportion of false-positives, a higher specificity, and may detect minute amounts of DNA from a dead organism. Furthermore, because the specimen type and gene target for MRSA detection are the same in adults and children, NAAT's accuracy in adult blood samples could provide a glimpse of its applicability for subsequent detection in the pediatric population. Therefore, NAAT should be considered as the preferred initial tests for diagnosing BSI in order to avoid unnecessary antiMRSA therapy. The utility of NAAT, in combination with microbiological culture, should be considered whenever feasible. Given the limited data on MRSA, it would be of interest to conduct a detailed investigation using a higher number of prospective studies to fully validate the clinical outcomes associated with NAAT's utility. Additionally, future research should analyze additional measures, including NAAT's impact on decreased hospitalizations, cost-effectiveness, antibiotic-related adverse effects, and electronic clinical decision supporting tools to accelerate therapy adjustment.

\section{DATA AVAILABILITY STATEMENT}

The original contributions presented in the study are included in the article/Supplementary Material, 
further inquiries can be directed to the FUNDING

corresponding author/s.

\section{AUTHOR CONTRIBUTIONS}

SCO conceptualized the study. KC, AAM, and SCO performed the literature search, analyzed data, and drafted the manuscript. Y-JS, SA, CS, C-LD, and SCO reviewed and edited the manuscript. All authors contributed to the article and approved the submitted version.

\section{REFERENCES}

1. Del Rio A, Cervera C, Moreno A, Moreillon P, Miró JM. Patients at risk of complications of staphylococcus aureus bloodstream infection. Clin Infect Dis. (2009) 48:S246-53. doi: 10.1086/598187

2. Fowler VG, Olsen MK, Corey GR, Woods CW, Cabell CH, Reller LB, et al. Clinical identifiers of complicated staphylococcus aureus bacteremia. Arch Intern Med. (2003) 163:2066-72. doi: 10.1001/archinte.163.17.2066

3. Wongboot W, Chomvarin C, Engchanil C, Namwat W, Chaimanee P. Application of SYBR green real-time quantitative PCR and conventional PCR for the detection of methicillin-resistant staphylococcus aureus in blood samples. Chiang Mai J Sci. (2016) 43:737-46. Available online at: http://epg. science.cmu.ac.th/ejournal/

4. Cattoir V, Merabet L, Djibo N, Rioux C, Legrand P, Girou E, et al. Clinical impact of a real-time PCR assay for rapid identification of staphylococcus aureus and determination of methicillin resistance from positive blood cultures. Clin Microbiol Infect. (2011) 17:42531. doi: 10.1111/j.1469-0691.2010.03233.x

5. Trindade PA, Pacheco RL, Costa SF, Rossi F, Barone AA, Mamizuka EM, et al. Prevalence of SCCmec type IV in nosocomial bloodstream isolates of methicillin-resistant staphylococcus aureus. J Clin Microbiol. (2005) 43:34357. doi: 10.1128/JCM.43.7.3435-3437.2005

6. Laupland KB, Lyytikäinen O, Sgaard M, Kennedy K, Knudsen JD, Ostergaard $\mathrm{C}$, et al. The changing epidemiology of staphylococcus aureus bloodstream infection: a multinational population-based surveillance study. Clin Microbiol Infect. (2013) 19:465-71. doi: 10.1111/j.1469-0691.2012.03903.x

7. Brown J, Paladino JA. Impact of rapid methicillin-resistant Staphylococcus aureus polymerase chain reaction testing on mortality and cost effectiveness in hospitalized patients with bacteraemia. Pharmacoeconomics. (2010) 28:56775. doi: 10.2165/11533020-000000000-00000

8. Song K-H, Kim ES, Sin H-Y, Park K-H, Jung S-I, Yoon N, et al. Characteristics of invasive staphylococcus aureus infections in three regions of Korea, 2009-2011: a multi-center cohort study. BMC Infect Dis. (2013) 13:581. doi: 10.1186/1471-2334-13-581

9. Kim C-J, Kim H-B, Oh M-d, Kim Y, Kim A, Oh S-H, et al. The burden of nosocomial staphylococcus aureus bloodstream infection in South Korea: a prospective hospital-based nationwide study. BMC Infect Dis. (2014) 14:590. doi: 10.1186/s12879-014-0590-4

10. Van Hal SJ, Jensen SO, Vaska VL, Espedido BA, Paterson DL, Gosbell IB. Predictors of mortality in staphylococcus aureus bacteremia. Clin Microbiol Rev. (2012) 25:6362-86. doi: 10.1128/CMR.05022-11

11. Cusumano JA, Dupper AC, Malik Y, Gavioli EM, Banga J, Berbel Caban A. Staphylococcus aureus bacteremia in patients infected with COVID19: a case series. Open Forum Infect Dis. (2020) 7:518. doi: 10.1093/ofid/ ofaa518

12. Liu C, Bayer A, Cosgrove SE, Daum RS, Fridkin SK, Gorwitz RJ, et al. Clinical practice guidelines by the infectious diseases Society of America for the treatment of methicillin-resistant staphylococcus aureus infections in adults and children. Clin Infect Dis. (2011) 52:e18-55. doi: 10.1093/cid/c iq146

13. Zhang S, Sun X, Chang W, Dai Y, Ma X. Systematic review and metaanalysis of the epidemiology of vancomycin-intermediate and heterogeneous
This study was funded by the Doctoral Research Fund of Affiliated Hospital of Southwest Medical University to SCO and KC.

\section{SUPPLEMENTARY MATERIAL}

The Supplementary Material for this article can be found online at: https://www.frontiersin.org/articles/10.3389/fped. 2021.713447/full\#supplementary-material

vancomycin-intermediate staphylococcus aureus isolates. PLoS ONE. (2015) 10:e0136082. doi: 10.1371/journal.pone.0136082

14. Stryjewski ME, Szczech LA, Benjamin Jr DK, Inrig JK, Kanafani ZA, Engemann JJ, et al. Use of vancomycin or first-generation cephalosporins for the treatment of hemodialysis-dependent patients with methicillinsusceptible staphylococcus aureus bacteremia. Clin Infect Dis. (2007) 44:1906. doi: 10.1086/510386

15. Martinez RM, Bauerle ER, Fang FC, Butler-Wu SM. Evaluation of three rapid diagnostic methods for direct identification of microorganisms in positive blood cultures. J Clin Microbiol. (2014) 52:2521-9. doi: 10.1128/JCM.00529-14

16. Reier-Nilsen T, Farstad T, Nakstad B, Lauvrak V, Steinbakk M. Comparison of broad range 16S rDNA PCR and conventional blood culture for diagnosis of sepsis in the newborn: a case control study. BMC Pediatr. (2009) 9:5. doi: 10.1186/1471-2431-9-5

17. Opota O, Croxatto A, Prod'hom G, Greub G. Blood culture-based diagnosis of bacteraemia: state of the art. Clin Microbiol Infect. (2015) 21:31322. doi: 10.1016/j.cmi.2015.01.003

18. Connell TG, Rele M, Cowley D, Buttery JP, Curtis N. How reliable is a negative blood culture result? Volume of blood submitted for culture in routine practice in a children's hospital. Pediatrics. (2007) 119:8916. doi: 10.1542/peds.2006-0440

19. Chiesa C, Panero A, Osborn JF, Simonetti AF, Pacifico L. Diagnosis of neonatal sepsis: a clinical and laboratory challenge. Clin Chem. (2004) 50:27987. doi: 10.1373/clinchem.2003.025171

20. McHugh MP, Parcell BJ, MacKenzie FM, Templeton KE. Rapid molecular testing for staphylococcus aureus bacteraemia improves clinical management. J Med Microbiol. (2020) 69:552-7. doi: 10.1099/jmm.0.001171

21. Makhoul IR, Smolkin T, Sujov P, Kassis I, Tamir A, Shalginov R, et al. PCRbased diagnosis of neonatal staphylococcal bacteremias. J Clin Microbiol. (2005) 43:4823-5. doi: 10.1128/JCM.43.9.4823-4825.2005

22. Ohlin A, Bäckman A, Björkqvist M, Mölling P, Jurstrand M, Schollin J. Realtime PCR of the 16S-rRNA gene in the diagnosis of neonatal bacteraemia. Acta Paediatr. (2008) 97:1376-80. doi: 10.1111/j.1651-2227.2008.00924.x

23. Wolk D, Struelens M, Pancholi P, Davis T, Della-Latta P, Fuller D, et al. Rapid detection of staphylococcus aureus and methicillin-resistant S. Aureus (MRSA) in wound specimens and blood cultures: multicenter preclinical evaluation of the Cepheid Xpert MRSA/SA skin and soft tissue and blood culture assays. J Clin Microbiol. (2009) 47:823-6. doi: 10.1128/JCM.01884-08

24. Baek Y-H, Jo M-Y, Song M-S, Hong S-B, Shin K-S. Application of loopmediated isothermal amplification (LAMP) assay to rapid detection of methicillin-resistant staphylococcus aureus from blood cultures. Biomed Sci Lett. (2019) 25:75-82. doi: 10.15616/BSL.2019.25.1.75

25. Salimnia H, Fairfax MR, Lephart PR, Schreckenberger P, DesJarlais SM, Johnson JK, et al. Evaluation of the FilmArray blood culture identification panel: results of a multicenter controlled trial. J Clin Microbiol. (2016) 54:68798. doi: 10.1128/JCM.01679-15

26. McInnes MD, Moher D, Thombs BD, McGrath TA, Bossuyt PM, Clifford T, et al. Preferred reporting items for a systematic review and meta-analysis of diagnostic test accuracy studies: the PRISMA-DTA statement. JAMA. (2018) 319:388-96. doi: 10.1001/jama.2017.19163

27. Whiting PF, Rutjes AW, Westwood ME, Mallett S, Deeks JJ, Reitsma JB, et al. QUADAS-2: a revised tool for the quality 
assessment of diagnostic accuracy studies. Ann Intern Med. (2011) 155:529-36. doi: 10.7326/0003-4819-155-8-201110180-00009

28. Cochrane T. Review Manager (RevMan) 5.4. Copenhagen: The Nordic Cochrane Centre. (2008). p. 373.

29. Zamora J, Abraira V, Muriel A, Khan K, Coomarasamy A. Meta-DiSc: a software for meta-analysis of test accuracy data. BMC Med Res Methodol. (2006) 6:31. doi: 10.1186/1471-2288-6-31

30. Higgins JP, Thomas J, Chandler J, Cumpston M, Li T, Page MJ, et al. Cochrane Handbook For Systematic Reviews of Interventions. Chichester: John Wiley \& Sons (2019).

31. Deeks JJ, Macaskill P, Irwig L. The performance of tests of publication bias and other sample size effects in systematic reviews of diagnostic test accuracy was assessed. J Clin Epidemiol. (2005) 58:882-93. doi: 10.1016/j.jclinepi.2005.01.016

32. Abd El-Aziz NK, Gharib AA, Mohamed EAA, Hussein AH. Real-time PCR versus MALDI-TOF MS and culture-based techniques for diagnosis of bloodstream and pyogenic infections in humans and animals. J Appl Microbiol. (2020) 130:1630-44. doi: 10.1111/jam.14862

33. Arabestani MR, Fazzeli H, Esfahani BN. Identification of the most common pathogenic bacteria in patients with suspected sepsis by multiplex PCR. $J$ Infect Dev Ctries. (2014) 8:461-8. doi: 10.3855/jidc.3856

34. Bloos F, Hinder F, Becker K, Sachse S, Dessap AM, Straube E, et al. A multicenter trial to compare blood culture with polymerase chain reaction in severe human sepsis. Intens Care Med. (2010) 36:2417. doi: 10.1007/s00134-009-1705-Z

35. Etchebarne BE, Li Z, Stedtfeld RD, Nicholas MC, Williams MR, Johnson TA, et al. Evaluation of nucleic acid isothermal amplification methods for human clinical microbial infection detection. Front Microbiol. (2017) 8:2211. doi: 10.3389/fmicb.2017.02211

36. Faraji R, Behjati-Ardakani M, Faraji N, Moshtaghioun SM, Kalantar SM, Pedarzadeh A, et al. Molecular diagnosis of bacterial definite infective endocarditis by real-time polymerase chain reaction. Cardiol Res. (2018) 9:99-106. doi: 10.14740/cr687w

37. Fernández-Romero N, Quiles I, Jiménez C, Oliva MOL, Rivas B, Mingorance J, et al. Use of multiplex PCR in diagnosis of bloodstream infections in kidney patients. Diagn Microbiol Infect Dis. (2014) 80:936. doi: 10.1016/j.diagmicrobio.2014.07.001

38. García-Gudiño I, Yllescas-Medrano E, Maida-Claros R, Soriano-Becerril D, Díaz NF, García-López G, et al. Microbiological comparison of blood culture and amplification of $16 \mathrm{~S}$ rDNA methods in combination with DGGE for detection of neonatal sepsis in blood samples. Eur J Pediatr. (2018) 177:8593. doi: 10.1007/s00431-017-3036-3

39. Ginn AN, Hazelton B, Shoma S, Cullen M, Solano T, Iredell JR. Quantitative multiplexed-tandem PCR for direct detection of bacteraemia in critically ill patients. Pathology. (2017) 49:304-8. doi: 10.1016/j.pathol.2016.1 0.014

40. Grosse-Onnebrink J, Stehling F, Tschiedel E, Olivier M, Mellies U, Schmidt $\mathrm{R}$, et al. Bacteraemia and fungaemia in cystic fibrosis patients with febrile pulmonary exacerbation: a prospective observational study. BMC Pulm Med. (2017) 17:96. doi: 10.1186/s12890-017-0440-4

41. Josefson P, Strålin K, Ohlin A, Ennefors T, Dragsten B, Andersson L, et al. Evaluation of a commercial multiplex PCR test (SeptiFast) in the etiological diagnosis of community-onset bloodstream infections. Eur J Clin Microbiol Infect Dis. (2011) 30:1127-34. doi: 10.1007/s10096-011-1 201-6

42. Kitagawa Y, Ueda M, Ando N, Endo M, Ishibiki K, Kobayashi Y, et al. Rapid diagnosis of methicillin-resistant staphylococcus aureus bacteremia by nested polymerase chain reaction. Ann Surg. (1996) 224:66571. doi: 10.1097/00000658-199611000-00010

43. Knabl L, Mutschlechner W, Orth-Höller D. Evaluation of a multiplex OnSpot primer-extension PCR assay in the diagnosis of sepsis. J Microbiol Methods. (2016) 120:91-3. doi: 10.1016/j.mimet.2015.12.001

44. Korber F, Zeller I, Grünstäudl M, Willinger B, Apfalter P, Hirschl $\mathrm{AM}$, et al. SeptiFast versus blood culture in clinical routine - a report on 3 years experience. Wien Klin Wochenschr. (2017) 129:42734. doi: 10.1007/s00508-017-1181-3

45. Lehmann LE, Alvarez J, Hunfeld KP, Goglio A, Kost GJ, Louie $\mathrm{RF}$, et al. Potential clinical utility of polymerase chain reaction in microbiological testing for sepsis. Crit Care Med. (2009) 37:3085-90. doi: 10.1097/CCM.0b013e3181b033d7

46. Liberto MC, Puccio R, Matera G, Lamberti AG, Quirino A, Barreca GS, et al. Applications of lightcycler staphylococcus M-GRADE assay to detect staphylococcus aureus and coagulase-negative staphylococci in clinical blood samples and in blood culture bottles. Infez Med. (2006) 14:71-6.

47. Liu CF, Shi XP, Chen Y, Jin Y, Zhang B. Rapid diagnosis of sepsis with TaqMan-Based multiplex real-time PCR. J Clin Lab Anal. (2018) 32:e22256. doi: 10.1002/jcla.22256

48. Lucignano B, Ranno S, Liesenfeld O, Pizzorno B, Putignani L, Bernaschi $\mathrm{P}$, et al. Multiplex PCR allows rapid and accurate diagnosis of bloodstream infections in newborns and children with suspected sepsis. J Clin Microbiol. (2011) 49:2252-58. doi: 10.1128/JCM.02460-10

49. Moore CC, Jacob ST, Banura P, Zhang J, Stroup S, Boulware DR, et al. Etiology of sepsis in Uganda using a quantitative polymerase chain reaction-based TaqMan Array Card. Clin Infect Dis. (2019) 68:26672. doi: $10.1093 /$ cid/ciy472

50. Obara H, Aikawa N, Hasegawa N, Hori S, Ikeda Y, Kobayashi Y, et al. The role of a real-time PCR technology for rapid detection and identification of bacterial and fungal pathogens in whole-blood samples. J Infect Chemother. (2011) 17:327-33. doi: 10.1007/s10156-010-0168-Z

51. Oeser C, Pond M, Butcher P, Bedford Russell A, Henneke P, Laing K, et al. PCR for the detection of pathogens in neonatal early onset sepsis. PLoS ONE. (2020) 15:e0226817. doi: 10.1371/journal.pone.0226817

52. Pasqualini L, Mencacci A, Leli C, Montagna P, Cardaccia A, Cenci E, et al. Diagnostic performance of a multiple real-time PCR assay in patients with suspected sepsis hospitalized in an internal medicine ward. J Clin Microbiol. (2012) 50:1285-1288. doi: 10.1128/JCM.06793-11

53. Peters RP, van Agtmael MA, Gierveld S, Danner SA, Groeneveld AB, Vandenbroucke-Grauls CM, et al. Quantitative detection of staphylococcus aureus and enterococcus faecalis DNA in blood to diagnose bacteremia in patients in the intensive care unit. J Clin Microbiol. (2007) 45:36416. doi: 10.1128/JCM.01056-07

54. Rogina P, Skvarc M, Stubljar D, Kofol R, Kaasch A. Diagnostic utility of broad range bacterial 16S rRNA gene PCR with degradation of human and free bacterial DNA in bloodstream infection is more sensitive than an inhouse developed PCR without degradation of human and free bacterial DNA. Mediators Inflamm. (2014) 2014:108592. doi: 10.1155/2014/108592

55. Santolaya ME, Farfán MJ, De La Maza V, Cociña M, Santelices F, Alvarez AM, et al. Diagnosis of bacteremia in febrile neutropenic episodes in children with cancer: microbiologic and molecular approach. Pediatr Infect Dis. (2011) 30:957-61. doi: 10.1097/INF.0b013e31822a37d7

56. Schaub N, Boldanova T, Noveanu M, Arenja N, Hermann H, Twerenbold $\mathrm{R}$, et al. Incremental value of multiplex real-time PCR for the early diagnosis of sepsis in the emergency department. Swiss Med Wkly. (2014) 144:w13911. doi: 10.4414/smw.2014.13911

57. van den Brand $M$, van den Dungen FAM, Bos MP, van Weissenbruch MM, van Furth AM, de Lange A, et al. Evaluation of a real-time PCR assay for detection and quantification of bacterial DNA directly in blood of preterm neonates with suspected late-onset sepsis. Crit Care. (2018) 22:105. doi: 10.1186/s13054-018-2010-4

58. Wallet F, Nseir S, Baumann L, Herwegh S, Sendid B, Boulo M, et al. Preliminary clinical study using a multiplex real-time PCR test for the detection of bacterial and fungal DNA directly in blood. Clin Microbiol Infect. (2010) 16:774-9. doi: 10.1111/j.1469-0691.2009.02940.x

59. Wu YD, Chen LH, Wu XJ, Shang SQ, Lou JT, Du LZ, et al. Gram stain-specific-probe-based real-time PCR for diagnosis and discrimination of bacterial neonatal sepsis. J Clin Microbiol. (2008) 46:2613-9. doi: 10.1128/JCM.02237-07

60. Xiao Y, Shen X, Zhao QF, Yao YH, Yang TC, Niu JJ. Evaluation of realtime PCR coupled with multiplex probe melting curve analysis for pathogen detection in patients with suspected bloodstream infections. Front Cell Infect Microbiol. (2019) 9:1-11. doi: 10.3389/fcimb.2019.00361

61. Yanagihara K, Kitagawa Y, Tomonaga M, Tsukasaki K, Kohno S, Seki M, et al. Evaluation of pathogen detection from clinical samples by real-time polymerase chain reaction using a sepsis pathogen DNA detection kit. Crit Care. (2010) 14:R159. doi: 10.1186/ cc9234 
62. Zboromyrska Y, De la Calle C, Soto M, Sampietro-Colom L, Soriano A, Alvarez-Martínez MJ, et al. Rapid diagnosis of staphylococcal catheter-related bacteraemia in direct blood samples by real-time PCR. PLoS ONE. (2016) 11:e0161684. doi: 10.1371/journal.pone.0161684

63. Ziegler I, Fagerstrom A, Strålin K, Molling P. Evaluation of a commercial multiplex PCR assay for detection of pathogen DNA in blood from patients with suspected sepsis. PLoS ONE. (2016) 11:e0167883. doi: 10.1371/journal.pone.0167883

64. Wang HY, Kim S, Kim J, Park SD, Uh Y, Lee H. Multiplex realtime PCR assay for rapid detection of methicillin-resistant staphylococci directly from positive blood cultures. J Clin Microbiol. (2014) 52:1911920. doi: 10.1128/JCM.00389-14

65. Qian QF, Eichelberger K, Kirby JE. Rapid identification of staphylococcus aureus directly from Bactec blood culture broth by the BinaxNOW S. aureus test. J Clin Microbiol. (2014) 52:319-20. doi: 10.1128/JCM.02291-13

66. Misawa Y, Yoshida A, Saito R, Yoshida H, Okuzumi K, Ito N, et al. Application of loop-mediated isothermal amplification technique to rapid and direct detection of methicillin-resistant staphylococcus aureus (MRSA) in blood cultures. J Infect Chemother. (2007) 13:13440. doi: 10.1007/s10156-007-0508-9

67. Kumar A, Ellis P, Arabi Y, Roberts D, Light B, Parrillo JE, et al. Initiation of inappropriate antimicrobial therapy results in a fivefold reduction of survival in human septic shock. Chest. (2009) 136:1237-48. doi: 10.1378/chest.09-0087

68. Rello J, Van Engelen T, Alp E, Calandra T, Cattoir V, Kern W, et al. Towards precision medicine in sepsis: a position paper from the European society of clinical microbiology and infectious diseases. Clin Microbiol Infect. (2018) 24:1264-72. doi: 10.1016/j.cmi.2018.03.011

69. Pammi M, Flores A, Versalovic J, Leeflang MM. Molecular assays for the diagnosis of sepsis in neonates. Cochrane Database Syst Rev. (2017) 2:CD011926. doi: 10.1002/14651858.CD011926.pub2

70. Su G, Fu Z, Hu L, Wang Y, Zhao Z, Yang W. 16 S ribosomal ribonucleic acid gene polymerase chain reaction in the diagnosis of bloodstream infections: a systematic review and meta-analysis. PLoS ONE. (2015) 10:e127195. doi: 10.1371/journal.pone.0127195

71. Chang S-S, Hsieh W-H, Liu T-S, Lee S-H, Wang C-H, Chou H-C, et al. Multiplex PCR system for rapid detection of pathogens in patients with presumed sepsis-a systemic review and meta-analysis. PLoS ONE. (2013) 8:e62323. doi: 10.1371/journal.pone.0062323

72. Altez-Fernandez C, Ortiz V, Mirzazadeh M, Zegarra L, Seas C, Ugarte-Gil C. Diagnostic accuracy of nucleic acid amplification tests (NAATs) in urine for genitourinary tuberculosis: a systematic review and meta-analysis. BMC Infect Dis. (2017) 17:390. doi: 10.1186/s12879-017-2476-8

73. Chen K, Ahmed S, Sun C, Sheng Y-J, Wu G, Deng C-L, et al. Molecular amplification assays accuracy for diagnosis of staphylococcal pneumonia: a systematic review and meta-analysis. J Clin Microbiol. (2021) 59:e0300320. doi: 10.1128/JCM.03003-20

Conflict of Interest: The authors declare that the research was conducted in the absence of any commercial or financial relationships that could be construed as a potential conflict of interest.

Publisher's Note: All claims expressed in this article are solely those of the authors and do not necessarily represent those of their affiliated organizations, or those of the publisher, the editors and the reviewers. Any product that may be evaluated in this article, or claim that may be made by its manufacturer, is not guaranteed or endorsed by the publisher.

Copyright (C) 2021 Chen, Malik, Sheng, Ahmed, Sun, Deng and Ojha. This is an open-access article distributed under the terms of the Creative Commons Attribution License (CC BY). The use, distribution or reproduction in other forums is permitted, provided the original author(s) and the copyright owner(s) are credited and that the original publication in this journal is cited, in accordance with accepted academic practice. No use, distribution or reproduction is permitted which does not comply with these terms. 